\title{
L'intégration des formats et le catalogage des publications en série
}

\section{Format Integration and the Cataloguing of Serials \\ La integración de los formatos y la catalogación de las publicaciones en serie}

\section{Wayne Jones et Young-Hee Queinnec}

Volume 40, numéro 3, juillet-septembre 1994

URI : https://id.erudit.org/iderudit/1033472ar

DOI : https://doi.org/10.7202/1033472ar

Aller au sommaire du numéro

Éditeur(s)

Association pour l'avancement des sciences et des techniques de la documentation (ASTED)

ISSN

0315-2340 (imprimé)

2291-8949 (numérique)

Découvrir la revue

Citer cet article

Jones, W. \& Queinnec, Y.-H. (1994). L'intégration des formats et le catalogage des publications en série. Documentation et bibliothèques, 40(3), 133-138. https://doi.org/10.7202/1033472ar
Résumé de l'article

Après un rappel de l'origine et de l'évolution du « Machine Readable Cataloguing " (MARC), les auteurs décrivent les résultats de l'intégration des formats bibliographiques et en évaluent l'effet sur le travail des catalogueurs de publications en série au Canada et aux États-Unis.
Tous droits réservés @ Association pour l'avancement des sciences et des techniques de la documentation (ASTED), 1994
Ce document est protégé par la loi sur le droit d'auteur. L'utilisation des services d'Érudit (y compris la reproduction) est assujettie à sa politique d'utilisation que vous pouvez consulter en ligne. 


\title{
L'intégration des formats et le catalogage des publications en série *
}

\author{
Wayne Jones \\ Chef de la Section des publications en série \\ Bibliothèque nationale du Canada \\ Young-Hee Queinnec \\ Chef du Bureau MARC canadien \\ Bibliothèque nationale du Canada
}

Après un rappel de l'origine et de l'évolution du "Machine Readable Cataloguing" (MARC), les auteurs décrivent les résultats de l'intégration des formats bibliographiques et en évaluent l'effet sur le travail des catalogueurs de publications en série au Canada et aux États-Unis.

\section{Format Integration and the Cataloguing of Serials}

The authors recall the origins and the development of Machine Readable Cataloguing (MARC). They discuss the results of the integration of bibliographic formats and evaluate their effect on the work of serial cataloguers in Canada and the United States.

\section{La integración de los formatos y la catalogación de las publicaciones en serie}

Después de la evocación delorigen y de la evolución de la Machine Readable Cataloguing (MARC), los autores describen los resultados de la integración de los formatos bibliográficos y evaluan los efectos de la MARC sobre el trabajo de los bibliotecarios responsables de la catalogación de las publicaciones en serie en Canadá y en los Estados Unidos.
Jadis, quand les collections d'une bibliothèque se composaient surtout de documents imprimés et que le catalogue était constitué de fiches classées avec grand soin dans des tiroirs, on n'avait guère besoin des formats normalisés pour l'enregistrement des données bibliographiques sur support informatique.

Le premier format utilisé pour le catalogage informatique (MARC, selon le terme anglais), c'est-à-dire un document établissant les critères de désignation des données bibliographiques, est le fruit d'un projet pilote mené par la Library of Congress à la fin des années 1960. Les formats MARC canadiens pour les monographies et les publications en série, produits respectivement en 1973 et 1974 , étaient fondés sur les recommandations du Groupe de travail sur MARC au Directeur général de la Bibliothèque nationale du Canada. Ce groupe a tout particulièrement recommandé de «réduire au minimum les divergences de ce format canadien d'avec celui du MARC II de la Library of Congress ${ }^{1}$. Cette exigence a été respectée chaque fois que l'on a révisé et amé- lioré les formats MARC au cours des années.

II en résulte que les formats MARC canadien et américain sont généralement compatibles. L'une des principales différences entre le CAN/MARC et I'USMARC vient de ce que le premier comporte des zones $9 X X$, réservées à l'inscription de l'équivalent français ou anglais d'un nom ou d'un titre uniforme. Ces zones sont destinées à permettre l'accès bibliographique et à recevoir les informations bibliographiques requises dans un pays bilingue comme le Canada. Abstraction faite de ces zones $9 \times X$ et de quelques particularités historiques mineures, le CAN/MARC et I'USMARC sont quasiment identiques.

Le format CAN/MARC pour les monographies, à l'instar du premier format LCMARC, était d'abord destiné exclusivement aux textes. Ses spécifications ont été élargies, au cours des années, afin qu'il puisse être utilisé aussi pour le catalogage de différents documents non textuels. En 1988, le format CAN/MARC pour les monographies comportait des spécifications pour les documents audiovisuels, la musique et les enregistrements sonores, les documents cartographiques et les microformes.

La fusion, en 1988, des formats pour monographies et pour publications en série, a donné le Format de communication du

\footnotetext{
* Cet article a paru d'abord dans The Serials Librarian, vol. 25, no. 1/2, 1994, c 1994 par Haworth Press, Inc. Tous droits réservés. Reproduction et traduction autorisées. Pour obtenir des exemplaires de ce texte en anglais, communiquez avec Marianne Arnold au Haworth Document Delivery Service (tél. 1-800-3-HAWORTH; 10 Alice St., Binghamton, NY 13904). Pour toute autre question touchant les droits ou les autorisations, communiquez avec Wanda Latour à l'adresse indiquée cidessus.
}

1. Groupe de travail sur MARC, MARC canadien: rapportd'activités et recommandations du Groupe de travail sur MARC concernant un format $M A R C$ canadien pour les monographies et un formatMARC canadien pourles publications en série soumis au Directeur général, Ottawa, Bibliothèque nationale du Canada, 1972, p. 3. 
MARC canadien: données bibliographiques. Cette version combinée comportait en outre des spécifications pour les fichiers informatiques et des spécifications à utiliser pour la description des documents d'archives. Aux États-Unis, les deux formats avaient été fusionnés et publiés en 1980 en tant que MARC Format for Bibliographic Data, dont une nouvelle version fut publiée en 1988 sous le titre USMARC Format for Bibliographic Data.

Les formats ont été fusionnés, certes, mais ils n'ont jamais été réellement intégrés. En fait, le format bibliographique CAN/MARC est plutôt un regroupement de formats différents, car il contient les particularités conservées des formats qui. datent de l'époque où les mêmes données étaient traitées différemment selon la nature des documents. Ainsi, pour les variantes du titre, la zone 246 est utilisée exclusivement pour les publications en série, alors que la zone 740 est utilisée pour tous les autres types de documents.

En outre, il n'existe aucune possibilité d'enregistrer correctement les informations codées relatives à des publications en série non textuelles. Ainsi, pour cataloguer un enregistrement sonore en série, le catalogueur doit déterminer si le document doit être considéré comme une publication en série ou comme un enregistrement sonore. II n'est pas possible de le cataloguer comme un enregistrement sonore tout en saisissant, sous forme codée, ses particularités de publication en série, ni vice-versa.

Donc, la proposition 88-1, qui établit les spécifications d'intégration des formats bibliographiques USMARC, a été approuvée par le Committee on Representation in Machine-Readable Form of Bibliographic Information (MARBI) de l'American Library Association, lors de séances tenues en janvier et juillet 1988. Pour ce qui est du CAN/MARC, les mêmes changements furent examinés par le Comité canadien sur le MARC puis approuvés en octobre 1988.

\section{Raison d'être de l'intégration des for-} mats

L'intégration des formats (IF) a deux grands objectifs. En premier lieu, elle unifie les différents formats en écartant la grande question de la validité et les multiples particularités issues de l'évolution des différents formats au cours des années. Autrement dit, l'IF assurera, en principe, la validité de n'importe quel désignateur de conten $u^{2}$ pour toute notice, dans la mesure où il sera approprié. II faut préciser que, I'IF accomplie, les possibilités d'utilisation de certains désignateurs de contenu demeureront minces, de par leur nature. Par exemple, la zone $\mathbf{3 1 0}$ (périodicité courante de la publication) ne pourra évidemment être utilisée que pour les documents présentant les caractéristiques d'une publication en série.

En second lieu, l'IF est destinée à permettre l'enregistrement de caractéristiques matérielles additionnelles de publications en série non textuelles ainsi que d'autres documents constitués de plus d'un type de matériel. Dans le document de la Library of Congress sur les conséquences de l'IF, on estime que l'IF permet de décrire les caractéristiques de publication en série de n'importe lequel de ces ouvrages ainsi que ses caractéristiques archivistiques, peu importe le support ou la forme du matériel ${ }^{3}$.

\section{Changements apportés au catalogage des publications en série}

Donc, lorsque l'IF sera chose faite, à quels changements le catalogueur de publications en série pourra-t-il s'attendre et en quoi ceux-ci influeront-ils sur son travail? Vous trouverez ci-après les sept changements ou catégories de changements que le catalogueur de publications en série se doit de connaître.

\section{1. Éléments de données de longueur fixe - Caractéristiques matérielles additionnel- les}

La zone 006 est ajoutée pour contenir de l'information codée sur les caractéristiques matérielles additionnelles d'un document. Les informations codées primaires continueront d'être enregistrées dans la zone 008 . La zone 006 sera utilisée pour l'enregistrement des caractéristiques des publications en série non textuelles ou pour l'enregistrement des caractéristiques physiques secondaires d'un document se présentant sous plus d'un support ou d'un format. Par exemple, dans le cas d'un fichier informatique en série, on doit utiliser la zone 008 pour enregistrer les caractéristiques du fichier informatique et la zone 006 pour enregistrer ses caractéristiques de publication en série. De même, dans le cas d'un enregistrement sonore musical (monographie) accompagné d'un livret, on doit utiliser la zone 008 pour enregistrer les caractéristiques de l'enregistrement sonore et la zone 006 pour les caractéristiques relatives au livret.

Avecl'IF, on disposera de septzones 006 : publication en série (PS), livre (LV), fichier informatique (FI), document cartographique (DC), document de genres multiples (DM), musique et enregistrement sonore (MES) et document visuel (DV). La zone 006DM servira principalement à un usage archivistique (DA).

Le code inscrit à la position 6 (genre de matériel) de la zone guide de la notice détermine les caractéristiques à enregistrer dans la zone 006 et celles qui doivent être inscrites dans la zone 008 . En principe, le catalogueur doit choisir un code pour le guide/6; ensuite, il choisit le code 008 qui correspond au code du guide/ 6 , puis la ou les zones 006 qui correspondent aux caractéristiques additionnelles du document (voir tableau 1).

À noter que, pour le texte imprimé (guide/6 = a), le code du guide/7 (niveau bibliographique) détermine la zone (008LV ou 008PS) à choisir.

Après avoir choisi une zone 008 appropriée, le catalogueur peut ajouter une ou plusieurs zones 006 afin de saisir des caractéristiques matérielles additionnelles sous forme codée. La zone $006 \mathrm{com}$ prend 18 caractères (positions 0 à 17). La position 0 correspond au code du guide/ 6 de la notice (sauf pour 006PS; voir cidessous), et les positions de caractère 1 à 17 sont identiques aux positions de caractère 18 à 34 de la zone 008 appropriée.

2. Les désignateurs de contenu (étiquettes, indicateurs et codes de sous-zone) indiquent des éléments de données et (ou) fournissent d'autres informations à leur sujet.

3. Format Integration and Its Effect on the USMARC Bibliographic Format, 1992 ed., Washington, Library of Congress, Cataloging Distribution Service, 1992, p. 5. 
Ces positions contiennent les informations particulières au document. Dans l'exemple de fichier informatique en série donné plus haut, la valeur du guide/ 6 est «m» pour fichier informatique, les caractéristiques du fichier informatique sont contenues dans la zone 008 de la notice; le code 006/0 est «s» pour publication en série et les codes 006/1-17 sont les mêmes que les codes 008/18-34 pour une publication en série. Autrement dit, la zone 006PS sert à enregistrer les caractéristiques de publication en série du fichier informatique.

Comme nous l'avons vu ci-haut, la première position (006/0) de la zone 006 correspond au code guide/ 6 de la notice, la seule exception à cette règle étant 006PS, pour laquelle le code provient du guide/7 (niveau bibliographique)(voir tableau 2).

Le code de la position de caractère 0 de la zone 006 détermine les codes à utiliser dans les positions suivantes. Les possibilités pour 006/0 et la nature de la zone 006 qui en résulte pour l'enregistrement des données dans le reste de la zone, sont données dans le tableau 3.

La zone 006 convient pour les publications en série non textuelles ainsi que pour les publications en série principalement textuelles mais comportant du matériel d'accompagnement non textuel. La zone 006PS permet de saisir les caractéristiques de publication en série des premières tandis que, pour les secondes, le choix de la zone 006 dépend de la nature précise du matériel d'accompagnement Donc, les publications en série strictement textuelles ne requièrent pas l'emploi de la zone 006, car les caractéristiques inhérentes à la publication en série sont codées dans la zone 008, comme d'habitude.

\section{Variantes du titre}

Les zones suivantes deviendront périmées et seront remplacées par la zone 246, qui servira pour toutes les
Tableau 1 : Liste des codes du guide/6 avec les codes 008 correspondants

$\begin{array}{lc}\text { Code du guide/6 } & \text { Zone } 008\end{array}$

\begin{tabular}{|c|c|c|}
\hline a & Texte imprimé (y compris en microforme) & $\begin{array}{l}\text { 008LV } \\
\text { 008PS }\end{array}$ \\
\hline c & Musique imprimée ou en microforme & DOBMES \\
\hline d & Musique manuscrite (y compris en microforme) & OOBMES \\
\hline e & Document cartographique imprimé ou en microforme & OOBDC \\
\hline & Document cartographique manuscrit (y compris en microforme) & OO8DC \\
\hline g & Matériel visionné par projection & 008DV \\
\hline & Enregistrement sonore non musical & OOBMES \\
\hline & Enregistrement sonore musical & OOBMES \\
\hline k & Document iconique en deux dimensions non projeté & OO8DV \\
\hline m & Fichier informatique & $008 \mathrm{FI}$ \\
\hline & Ensemble multi-supports & 008DV \\
\hline & Document de genres multiples & 008DM \\
\hline & Artefact en trois dimensions & 008DV \\
\hline & Texte manuscrit (y compris en microforme) & $008 \mathrm{LV}$ \\
\hline
\end{tabular}

Tableau 2 : La zone 006 pour les publications en série se présente comme suit :
O06SE

Position de caractère

Type de 006
Périodicité
Régularité
Centre de l'ISSN
Type de publication en série
Support matériel du document original
Support matériel du document
Nature de l'ouvrage entier
Nature du contenu
Publication officielle
Publication de conférence
Blancs
Alphabet original du titre
Vedette du dernier titre/
titre successif

Type de 006

2 Régularité

3 Centre de l'ISSN

$4 \quad$ Type de publication en série

5 Support matériel du document original

6 Support matériel du document

$7 \quad$ Nature de l'ouvrage entier

8-10 Nature du contenu

11 Publication officielle

12 Publication de conférence

13-15 Blancs

$16 \quad$ Alphabet original du titre

17 Vedette du dernier titre titre successif
1 Périodicité
Correspond à

guide $/ 7$

008PS/18

008PS $/ 19$

008PS/20

008PS/21

O08PS/22

008PS/23

008PS/24

008PS/25-27

$008 \mathrm{PS} / 28$

008PS $/ 29$

008PS/33

008PS/34
Tableau 3

$006 / 0$

Zone 006 appropriée

a Texte imprimé (y compris en microforme)

OO6LV

Musique imprimée ou en microforme

O06MES

Musique manuscrite (y compris en microforme)

OO6MES

Document cartographique imprimé ou en microforme

O06DC

O06DC

Matériel visionné par projection

Enregistrement sonore non musical

OO6DV

O06MES

Enregistrement sonore musical

O06MES

OO6DV

Document iconique en deux dimensions non projeté

O06FI

Finformatique

OO6DV

Document de genres multiples

O06DM

Artefact en trois dimensions

OO6DV

Publication en série

O06PS

Texte manuscrit (y compris en microforme) 
variantes du titre, sans égard à la forme du matériel :

\section{zone 211 (acronyme ou titre abrégé) zone 212 (variante du titre) \\ zone 214 (titre augmenté)}

Les titres actuellement inscrits dans ceszones seront inscrits dans lazone 246, lorsque l'IF sera chose faite.

Les deux indicateurs de la zone 246 seront redéfinis, et deux nouvelles souszones (\$i et \$5) seront ajoutées. La zone 246 qui en résultera servira à l'inscription des variantes du titre qui figurent sur le document et à l'inscription de celles qui n'y figurent pas.

Les valeurs du premier indicateur seront les suivantes:

$0 \quad$ Note, pas de titre en rappel

1 Note, titre en rappel

2 Pas de note, pas de titre en rappel

3 Pas de note, titre en rappel

Pour les publications en série, le premier indicateur est actuellement utilisé uniquement pour indiquer si une entrée secondaire au titre doit ou non être faite. Après I'IF, cet indicateur servira également à produire une note. Les valeurs 0 et 1 sont donc redéfinies et les valeurs 2 et 3 sont nouvelles pour les publications en série.

Les valeurs du deuxième indicateur seront les suivantes:

b Aucune indication fournie

0 Partie du titre

1 Titre parallèle

2 Titre distinct

3 Autre titre

4 Titre de la couverture

5 Titre de la page de titre additionnelle

6 Titre de départ

7 Titre courant

8 Titre au dos

La valeur $\measuredangle$ sert pour les titres qui ne correspondent à aucune autre valeur de deuxième indicateur, par exemple les titres fournis par le catalogueur et qui ne figurent pas sur le document. La valeur $b$ peut aussi être utilisée avecla sous-zone \$i (texte d'affichage) pour produire une note avec un texte d'affichage spécial. La valeur $\not$ est la seule nouvelle valeur de la zone 246; toutes les autres valeurs demeurent inchangées. Étant donné que $b$ est utilisée pour les titres ne figurant pas sur le document, la valeur 3 est utilisée uniquement pour les titres qui figurent sur le document mais qui ne correspondent à aucune autre valeur.

La sous-zone $\$ i$ contient un texte d'affichage à utiliser dans une note. Le premier indicateur est mis à 0 ou 1 (imprimer une note) et le texte à afficher est entré dans la sous-zone \$i. Par exemple :

[245] 10\$aComputerized engineering index

[246] 16\$iAlso known as:\$aCOMPENDEX

Affiché dans une note comme suit : Also known as: COMPENDEX

[245] $00 \$ a 1+1$

[246] 1 $6 \$$ iVariante du titre:\$aUn plus un

Dans le premier exemple, le titre de la sous-zone \$a de la zone 246 est actuellement inscrit dans la zone 211 (acronyme ou titre abrégé) et, dans le deuxième exemple, le titre est inscrit dans la zone 212 (variante du titre).

Malgré les changements apportés à la zone 246 , il peut y avoir des cas (probablement rares en ce qui concerne le catalogage des publications en série) où il est nécessaire d'utiliser la zone 246 pour une entrée secondaire et d'employer une autre zone pour produire une note :

[246] 34\$aFair American

[500] $\nvdash \forall \$ a T i t r e$ de la couverture: The fair American

La sous-zone $\$ 5$ doit contenir le symbole de l'établissement qui détient l'exemplaire auquel s'applique la zone, ce qui permet d'indiquer les titres requis exclusivement par l'établissement local.

\section{Informations sur les acquisitions et les} abonnements

Les zones 265 (source d'acquisition/ adresse d'abonnement) et 350 (prix d'abonnement), qui servent actuellement à saisir les données sur l'acquisition et l'abonnement et le prix de l'abonnement ou les conditions en vertu desquelles la publication en série est disponible, deviendront périmées et seront remplacées par la zone 037. La zone 037 (numéro de stock) sera rebaptisée "source d'acquisition». La sous-zone \$b de la zone 037 comportera les informations sur l'acquisition et l'abonnement qui étaient auparavant inscrites dans la zone 265; la sous-zone \$c comportera le prix de l'abonnement ou les conditions en vertu desquelles la publication en série est disponible.

[037] ظ6\$bCanadian Institute of International Affairs, 150 King's College Circle, Toronto, Ont. M5S 2V9 $\$ c \$ 25.00$

[037] b6\$aPB-363547\$bNTIS\$fpaper copy $\$ c \$ 4.00 \$$ fmicrofiche $\$ c \$ 3.00$

\section{Périodicité et régularité des fichiers in- formatiques}

Dans le cas des fichiers informatiques qui sont des publications en série, le catalogueur n'utilisera plus 008FI/18-19 pour enregistrer la périodicité et la régularité. Ces positions deviendront périmées puisque ces caractéristiques (parmi d'autres) de publication en série pourront être saisies intégralement dans la zone 006PS. Étant donné que les zones 006 ont leur correspondance en 008, les positions de caractère 1 et 2 de $006 \mathrm{FI}$ ne seront pas définies. La zone 315 (périodicité) deviendra également périmée; on utilisera plutôt les zones 310 et 321 pour les fichiers informatiques, au même titre que pour n'importe quelle autre publication en série.

\section{Vedettes alternatives et secondaires}

Les valeurs 0 (vedette alternative) et 1 (vedette secondaire) du deuxième indicateur dans les zones $700,710,711$ et 740 deviendront périmées. La valeur 2 (vedette analytique) sera la seule à demeurer valide. Dans tous les cas sauf celui d'une vedette analytique, on devra utiliser la nouvelle valeur $b$ (aucune information fournie). Ainsi, le catalogueur n'aura plus à faire la distinction entre les vedettes alternatives et les vedettes secondaires, une tâche qui prend du temps.

\section{Titre analytique, associé ou non contrôlé}

Comme la zone 246 redéfinie permettra l'enregistrement de la plupart des variantes du titre, la zone 740 sera révisée et réservée aux titres de documents 
associés qui ne peuvent être portés ni dans la zone 246 ni dans les zones de liaison $(76 X-78 X)$. La zone 740 sera très peu utilisée pour autre chose que les titres analytiques.

\section{Changements déjà faits par la mise en oeuvre anticipée}

Certains changements, issus de l'IF, ont déjà été apportés au format bibliographique MARC. Au $1^{\text {or }}$ septembre 1991, certains désignateurs de contenu sont devenus périmés par suite de ce que l'on désigne par la mise en oeuvre anticipée de I'IF. Ces changements ont été examinés puis approuvés par la BNC, la LC et les grands réseaux (OCLC, RLIN, UTLAS et WLN).
Les désignateurs de contenu selectionnés lors de la mise en oeuvre anticipée sont ceux qui sont devenus périmés dans le cadre de l'IF. Les zones et sous-zones correspondantes ne sont plus utilisées, et les positions d'indicateur et de zone fixe sont laissées en blanc dans toutes les notices créées depuis le $1^{\text {er }}$ septembre 1991 Cependant, tous ces désignateurs de contenu demeurent valides pour les notices antérieures.

Vous trouverez ci-dessous les désignateurs de contenu visés par la mise en oeuvre anticipée de l'IF. Les symboles entre parenthèses renvoient au genre de matériel visé par l'élément. Là où des sous-zones ou des indicateurs sont indi-

\section{Tableau 4}

\begin{tabular}{|c|c|}
\hline Zone 008/30 & Code de page de titre disponible (PS) \\
\hline Zone 008/31 & Code d'index disponible (PS) \\
\hline Zone 008/32 & Code d'index récapitulatif disponible (PS) \\
\hline Zone 008/32 & Indicateur de vedette principale figurant dans la notice (LV MES DV) \\
\hline Zone $100-130$ & $\begin{array}{l}\text { Vedette principale (LV MES PS) } \\
\text { Second indicateur }\end{array}$ \\
\hline Zone 222 & $\begin{array}{l}\text { Titre-clé (PS FI) } \\
\quad \text { Premier indicateur }\end{array}$ \\
\hline Zone 260 & $\begin{array}{l}\text { Publication, diffusion, etc.. } \\
\text { Premier indicateur (LV DC MES PS) } \\
\text { Second indicateur (PS) }\end{array}$ \\
\hline Zone 302 & Pagination (LV) \\
\hline Zone 308 & Description matérielle des collections de films d'archives (DV) \\
\hline Zone 500 & $\begin{array}{l}\text { Notes générales } \\
\text { Sous-zone \$1 - Cote de la LC (DA PS) } \\
\text { Sous-zone \$x - ISSN (PS) } \\
\text { Sous-zone \$z - Source de la note (DA PS) }\end{array}$ \\
\hline
\end{tabular}

Zone 512

Zone 515

Zone 520

Volumes catalogués séparément avant ou après une date donnée (PS) Notes sur les particularités de la numérotation (PS)

Sous-zone $\$ z$ - Source de la note

Note de sommaire, etc.

Sous-zone $\$ z$ - Source de la note (DA LV FI PS)

Zone 525

Zone 527

Zone 530

Zone 546

Zone 547

Zone 550

Zone 570

Zone 580

Note de supplément (PS)

Sous-zone $\$ z$ - Source

Note de censure (DV)

Notes des formes matérielles additionnelles disponibles

Sous-zone $\$ z$ - Source de la note (DA FI PS DV)

Note sur les langues

Sous-zone $\$ z$ - Source de la note (PS)

Note explicative du titre antérieur (PS)

Sous-zone $\$ z$ - Source de la note

Note sur les organismes de publication (PS)

Premier indicateur

Sous-zone $\$ z$ - Source de la note

Note sur l'éditeur (PS)

Note de liaison complexe des vedettes (TOUS)

Sous-zone $\$ z$ - Source de la note qués pour une zone, seuls les indicateurs ou les sous-zones deviennent périmés; la zone demeure valide (voir tableau 4).

\section{Mise en oeuvre nationale de I'IF}

Depuis que les changements visés par l'IF ont été approuvés par le MARBI et le CCM, le groupe de mise en oeuvre del'IF - qui comporte des représentants de la LC, dela BNC, de l'ISM (autrefois UTLAS), de l'OCLC, du RLIN et du WLN - se réunit régulièrement au cours des conférences annuelles et des assemblées d'hiver de I'ALA. Le but du groupe est de coordonner la réalisation de l'IF au sein des différents établissements représentés.

En juin 1993, la LC a annoncé que la mise en oeuvre de l'IF serait reportée; elle proposait que la réalisation intégrale soit faite fin 1995 au lieu de début 1994. Par la suite, le groupe chargé de la mise en oeuvre de l'IF accepta la suggestion du RLIN de procéder en deux temps: les ajouts et les changements apportés aux zones variables $(010-8 X X)$ seront effectués avant la fin de 1994 et les ajouts et les changements apportés au guide et aux zones fixes $(006,007$ et 008) seront effectués avant la fin de 1995.

En prévision de la mise en oeuvre, de nouvelles éditions (en français et en anglais) du format bibliographique CAN/ MARC ont été publiées. Les changements visés par l'IF et les autres changements approuvés depuis janvier 1992 sont intégrés aux nouvelles versions que l'on a publiées en janvier 1994. Aux États-Unis, une nouvelle édition du format - comportant les zones variables intégrées - sera publiée au début de 1994.

\section{Mise en oeuvre locale de l'IF}

Rien n'empêche les bibliothèques de tenir compte des changements occasionnés par l'IF et de les incorporer à leur système local, dans la mesure où elles n'ont pas l'intention de diffuser les notices révisées avant la date prévue de mise en oeuvre.

En grande partie, ce seront les principes et les pratiques de catalogage de chaque établissement qui détermineront si les nouvelles zones disponibles doivent ou non être utilisées. Par exemple, même 
s'il est maintenant possible d'utiliser la zone 006PS pour la notice monographique d'une publication à feuilles mobiles afin de saisir les caractéristiques de publication en série ou de ses mises à jour, toutes les bibliothèques ne veulent ni ne peuvent se permettre d'adopter cette pratique. Toutefois, n'importe quel établissement qui reçoit des notices d'organismes nationaux de catalogage (ou d'autres établissements déjà passés à l'IF) devra être en mesure d'utiliser la zone 006 et d'intégrer les autres ajouts et changements visés par l'IF.

\section{Conclusion}

Grâce à l'intégration des formats, les divers formats du MARC sont unifiés et simplifiés, et il devient beaucoup plus facile d'enregistrer les caractéristiques matérielles additionnelles d'un document. Lorsque le désignateur de contenu est valide et approprié pour n'importe quel genre de matériel, il revient à l'utilisateur de choisir les zones qui conviennent, parmi l'ensemble des zones MARC disponibles. Les notices ainsi créées décrivent mieux la variété des documents bibliographiques que les bibliothèques et les établissements de catalogage doivent traiter. En fait, il s'agit du principal avantage de I'IF : possibilités plus vastes d'enregistrement des données des publications en série non textuelles.

\section{SwetScan}

\section{Un nouveau service de l'ICIST}
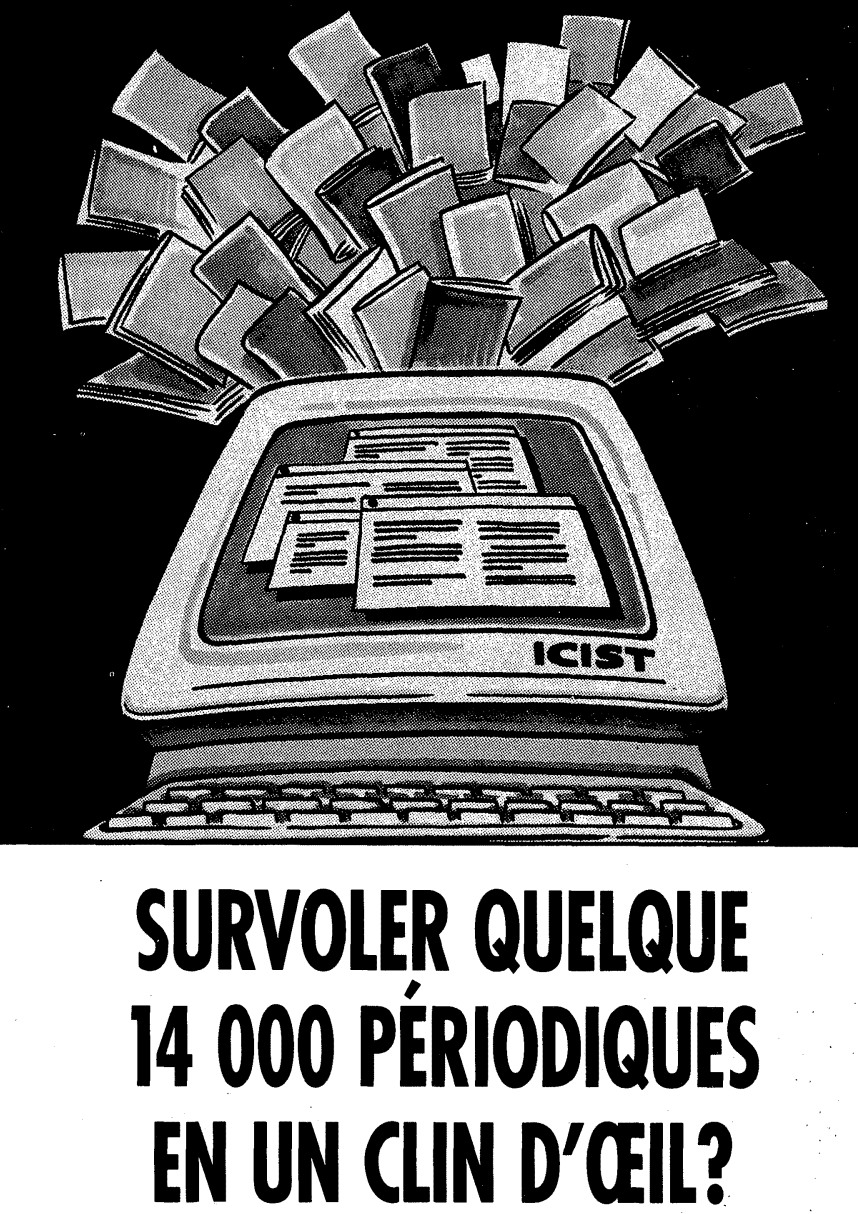

\section{Grâce à SwetScan, rien de plus simple!}

SwetScan est un service innovateur de l'ICIST qui vous offre l'accès aux tables des matières de quelque 14000 périodiques, et ce dans d'innombrables domaines.

Pourquoi payer pour de coûteux abonnements? Il vous suffit d'accéder à la base de données SwetScan et de sélectionner les périodiques que vous désirez consulter! La base de données est mise à jour de façon hebdomadaire.

Vous disposez d'un ordinateur ou d'un terminal équipé d'un modem ou encore vous avez accès à Internet? Vous n'avez besoin de rien de plus pour découvrir les trésors d'information que recèle SwetScan.

Pour obtenir de la documentation sur SwetScan, communiquez avec 1'ICIST par téléphone, au 1-800-668-1222, par télécopieur, au (613) 952-8244, à cisti.swetscan@nrc.ca

Gric-nRc 